\title{
Civilisations
}

Revue internationale d'anthropologie et de sciences

humaines

62 | 2013

Identité, culture et intimité

\section{Dis-moi quelle est la couleur de ta peau et je te dirai qui tu es}

Phénotype, langage et stéréotypes en République dominicaine

\section{Catherine Bourgeois}

\section{(2) OpenEdition}

Journals

Édition électronique

URL : http://journals.openedition.org/civilisations/3335

DOI : $10.4000 /$ civilisations.3335

ISSN : 2032-0442

Éditeur

Institut de sociologie de l'Université Libre de Bruxelles

\section{Édition imprimée}

Date de publication : 31 décembre 2013

Pagination : $31-50$

ISBN : 2-87263-042-2

ISSN : 0009-8140

Référence électronique

Catherine Bourgeois, "Dis-moi quelle est la couleur de ta peau et je te dirai qui tu es », Civilisations [En ligne], 62 | 2013, mis en ligne le 31 décembre 2016, consulté le 04 mai 2019. URL : http://

journals.openedition.org/civilisations/3335; DOI : 10.4000/civilisations.3335 


\title{
Dis-moi quelle est la couleur de ta peau et je te dirai qui tu es Phénotype, langage et stéréotypes en République dominicaine
}

\author{
Catherine BOURGEOIS
}

\begin{abstract}
Résumé : La République dominicaine porte encore les traces de la période coloniale, particulièrement en ce qui concerne l'usage, toujours actuel, d'un vocabulaire coloriste destiné à identifier et distinguer les personnes les unes par rapport aux autres. Ce riche vocabulaire est utilisé dans différents registres : sphère administrative mais aussi descriptions physiques des individus, interpellations, expressions langagières, etc. Dans de nombreux cas, les termes de couleur sont assortis de stéréotypes relatifs à l'identité sociale des individus, jugements qui interviennent parfois dans le déroulement des interactions. Cet article s'attache dans un premier temps à décrire le vocabulaire coloriste, ses "valeurs " et son lien avec la question haïtienne. Ensuite, il interroge les mécanismes de transmission et d'apprentissage des stéréotypes associés aux différents phénotypes à travers l'analyse de plusieurs médiateurs (termes d'adresse, injonctions sociales et bavardages, iconographie et scènes de la vie quotidienne) qui participent à la reproduction des catégories socio-raciales. Enfin, il se penche sur les stratégies sociales mises en ouvre pour occulter le stigmate de la couleur noire.
\end{abstract}

Mots-clés : catégories socio-raciales, apprentissage, stéréotypes, République dominicaine, relations dominico-haïtiennes.

\begin{abstract}
The Dominican Republic still bears traces of the colonial period which are particularly prominent in the daily use of a coloristic vocabulary designed to identify and distinguish people from each other. This rich vocabulary is used in different registers: administrative sphere but also physical descriptions of individuals, inquiries, verbal expressions, etc. In many cases, terms of colors are linked with stereotypes about the social identity of individuals. These judgments are sometimes involved in interactions. The first aim of this article is to describe the coloristic vocabulary, its "values" and its relationship with the Haitian issue. Then, it deals with the mechanisms of transmission and learning of the stereotypes associated with different phenotypes. This point is achieved through the analysis of several figures of speech and representations (greetings, gossip and social injunctions, iconography and scenes of everyday life) involved in the perpetuation of socio-racial categories. Finally, the author examines the social strategies implemented to hide the stigma of the black color.
\end{abstract}

Keywords: socio-racial categories, learning, stereotypes, Dominican Republic, DominicanHaitian relationships. 


\section{Introduction}

Juin 2004. Fraîchement descendue de l'avion, je déambule avec un ami dans les rues de Santo Domingo. Un homme me hèle : "Psst, hé ! Rubia [blonde] ! ». Les nouveaux arrivants ne faisant pas vraiment « couleur locale» font tous l'expérience de ces interpellations par des termes coloristes qui sont, je l'ai appris par la suite, connotés socialement et économiquement. Cette association « couleur-statut social » ne relevait pas tant de ma condition d'étrangère que du passé esclavagiste d'un pays où la couleur constitue un principe d'ordonnancement de la société.

Cet article se base sur des enquêtes de terrain menées en République dominicaine depuis 2004. Les vignettes ethnographiques présentées ci-dessous sont issues d'entretiens ${ }^{1}$ et d'observations réalisés dans deux régions du pays : la capitale, Santo Domingo, et la campagne de la région frontalière Nord (Dajabón, Loma de Cabrera, Restauración et Valle Nuevo). La recherche menée en 2004 à Santo Domingo portait sur les représentations du métissage auprès de la gent féminine de deux quartiers populaires de la ville et permit de soulever la question des relations dominico-haïtiennes. Ces relations sont souvent tendues voire conflictuelles, comme en témoignent les très nombreux articles de presse ainsi que les rapports internationaux portant sur les différentes formes de discrimination que subissent les Haïtiens et Dominico-Haïtiens en République dominicaine. Mon travail actuel porte sur ces relations à la frontière entre les deux pays. En m'intéressant à celles-ci, la question des stéréotypes ${ }^{2}$ raciaux et de leur transmission m'est à nouveau apparue incontournable.

Cet article interroge la reproduction, la transmission et l'apprentissage des stéréotypes dans cet espace insulaire. Pour ce faire, je m'attacherai d'abord à la genèse et à la description des catégories socio-raciales ainsi qu'à leurs usages actuels. Ensuite, je me pencherai sur les différents médiateurs transmettant les stéréotypes liés au vocabulaire coloriste. Enfin, je terminerai en expliquant et décrivant les stratégies permettant aux individus de limiter l'accès aux stigmates qu'ils portent, à travers la notion de couverture développée par Goffman (1975).

1 Bien que les recherches ethnographiques aient été menées également avec des Haïtiens, les personnes auxquelles il sera fait référence ici sont de nationalité dominicaine et les extraits présentés sont issus d'entretiens enregistrés mais aussi de conversations informelles qui se sont déroulés en espagnol et en créole avant d'être traduits en français par moi-même.

2 Dans ce travail, à l'instar d'Elisabeth Cunin, j'entendrai par stéréotypes « la réduction d'une identité ou d'une représentation à quelques traits simplifiés, aux connotations négatives ou, au moins, infériorisantes, obéissant à une logique essentialisante (les éléments ne sont liés ni au contexte, ni à l'histoire, ni aux interactions) et catégorielle (les individus appartenant à une catégorie étant immédiatement dotés de tous les attributs définissant cette catégorie) » (Cunin $2003: 241)$. 


\section{Retour sur la question raciale et les couleurs}

La question raciale ${ }^{3}$ en Amérique Latine et dans la Caraïbe apparut avec l'arrivée des colonisateurs européens et grandit avec l'implantation de l'esclavage dès $1520^{4}$. A cette époque, les relations de pouvoir étaient marquées par des critères (sexe, âge et force de travail, entre autres) jouant un rôle dans la stratification sociale (Quijano 2000 : 373). La rencontre avec les populations locales induisit un nouveau critère de différenciation juridique, et partant, de classification entre les groupes en présence : la couleur de la peau et par extension, le phénotype. La société coloniale s'organisa sur base d'une stratification sociale s'appuyant elle-même sur une première classification raciale ${ }^{5}$, bientôt « revue et augmentée » avec l'apparition de la figure du métis. Afin de réguler juridiquement la place de chacun dans la société, la logique de la « ligne de couleur » (Benoist et Bonniol 1994 : 60), soit « la stricte séparation installée entre les blancs et tous les autres » en vertu de laquelle les métis allaient être assimilés aux noirs (Bonniol $2006: 4$ ), fut mise en place suivie quelques temps après par un système juridique, le Code Noir, réglementant la vie et le sort des esclaves (Lucena Salmoral 1996).

Ainsi furent « créées » différentes catégories reflétant les degrés de décoloration qui, à leur tour, refléteraient aussi des relations de statuts :

La valorisation sociale de l'homme était plus élevée en l'absence de relations consanguines avec les esclaves noirs. Un blanc espagnol acquérait un statut social supérieur aux groupes susmentionnés [Liberto, Grifo, Mestizo, Tercerón et Cuarterón] indépendamment de sa position économique immédiate. (Franco $1970: 29)$

On retrouve cela dans la Cédule Royale signée à Valladolid le 29 avril 1549 présentant la hiérarchie socio-raciale suivante : a) Negros (esclaves noirs), b) Libertos

3 Si aujourd'hui chacun s'accorde pour dire que les races n'existent pas, scientifiquement parlant, il est cependant nécessaire de revenir sur ce terme (et ses dérivés) qui apparaîtront régulièrement dans ce texte. Le mot race ici employé ne renvoie nullement à une catégorie scientifique ou analytique, mais bien à une « catégorie pratique de l'appartenance » (selon l'expression de Cunin, 2003). En ce sens, l'objet auquel renvoie le terme race « devient réalité par le seul fait de sa désignation, donnant naissance à des pratiques sociales qui, elles, existent bien » (Cunin 2003 : 240). Par ailleurs, si le mot race apparaît ci-dessous dans des extraits d'entretiens, il ne sera que la traduction littérale du terme raza dont le sens dans la langue castillane ne recouvre pas l'acception que nous lui conférons dans la langue française. Dans le langage courant, les gens emploient raza pour parler du groupe social d'un individu en se référant à la couleur de la peau mais sans connotation péjorative.

4 Les premiers esclaves arrivèrent à Santo Domingo en 1503 mais étaient uniquement destinés au travail domestique. Ce n'est qu'à partir de 1520, avec l'expansion du commerce sucrier et la diminution de la population tainos soumise au travail forcé, que fut autorisé, par la Couronne espagnole, le commerce d'esclaves à destination des plantations (Moya Pons 2009 : 65).

5 Celle-ci distinguait notamment entre les esclaves ladinos et bozales (Deive 1979 : 297). Si aujourd'hui le ladino désigne communément la langue judéo-espagnole, le terme désignait au départ une langue romane proche du castillan parlée dans l'Andalousie musulmane du $9^{\text {eme }}$ siècle (Wright 1993 : 62); par extension, ce terme fut appliqué dans la péninsule aux esclaves noirs christianisés et parlant l'espagnol. Ceux-ci voyagèrent très tôt vers les Amériques comme domestiques. Ce terme continua à être employé dans les colonies afin de distinguer les ladinos des bozales amenés directement d'Afrique par les négriers et n'ayant pas eu (ou très peu) de contact avec les populations européennes (Schmidt $2003: 69$ ). 
(noirs ou mulâtres sortis du système esclavagiste), c) Mulatos (enfants de noirs et de blancs), d) Grifos (enfants d'indiens et de noirs), e) Mestizos (enfants d'indiens et de blancs), f) Tercerones (enfants de mulâtres et de blancs), g) Cuarterones (enfants de tercerones et de blancs) et enfin, h) Blancos (Espagnols de souche et descendants de blancs) (Franco 1970: 29 pour les catégories b, d, e, f et g).

La couleur en vint donc à constituer un critère de classement dans les sociétés esclavagistes et bientôt, l'équation « esclave = noir » se renversa justifiant ainsi les différences de statut social et le cantonnement des esclaves et de leurs descendants dans les strates les plus basses de la société (Bonniol 1992 : 55). Mais revenons à l'éventail des couleurs et phénotypes.

Dans ce type de taxinomie coloniale, la couleur de la peau n'était pas le seul critère physionomique à intervenir dans la catégorisation d'un individu. D'autres éléments comme les traits du visage (nez et bouche principalement) ainsi que le type de cheveux ${ }^{6}$ venaient compléter chaque phénotype reliant le blanc et le noir. La combinaison de ces traits avec la couleur de la peau a permis d'élaborer les phénotypes intermédiaires décrits actuellement en République dominicaine par les termes suivants ${ }^{7}$ (Bourgeois $2005: 63)$ :

\begin{tabular}{|c|c|}
\hline Azul & Noir très foncé, tirant sur le bleu \\
\hline Haitiano & Haïtien \\
\hline Prieto & Noir foncé \\
\hline $\begin{array}{l}\text { Negro abisinio/ } \\
\text { cenizo/retinto }\end{array}$ & Noir très foncé, couleur des cendres (ceniza) \\
\hline Negro & Noir \\
\hline Moreno & Brun proche du noir \\
\hline Moreno limpio & Brun un peu plus clair, « propre» \\
\hline Mulato & Mulâtre \\
\hline Trigueño & Mulâtre proche de la couleur du blé (trigo) \\
\hline Indio oscuro & Indien foncé \\
\hline Chocolate & Chocolat \\
\hline Indio canela & $\begin{array}{l}\text { Indien cannelle : peau chocolat,cheveux } \\
\text { lisses, traits « réguliers » }\end{array}$ \\
\hline Indio & littéralement : indien: mulâtre clair \\
\hline Indio claro & $\begin{array}{l}\text { Indien clair, couleur proche du blanc, } \\
\text { cheveux lisses et traits « fins » }\end{array}$ \\
\hline Indio lavao & littéralement : indien lavé, proche de indio claro \\
\hline
\end{tabular}

6 Lors de ma recherche ethnographique, j'ai relevé d'autres termes désignant le type de nez et de bouche : ñata, fino, ordinario, chata, lavado, narizona, nariz bombona, cacú, cacona. Les termes désignant la texture des cheveux sont: duro, muerto, greña, kinki/kinke, crespo, malo, rizado, ondulado, bueno, lacio (Bourgeois $2005: 64-65$ ).

7 Notons que cette taxinomie, principalement composée de termes coloristes, comporte aussi des termes raciaux comme haitiano, indio et gringo. Les deux premiers sont cependant régulièrement utilisés comme des catégories phénotypiques par les gens, principalement lorsqu'on les interroge sur les différentes couleurs disponibles pour décrire les individus. 


\begin{tabular}{|l|l|}
\hline Indio quemado & $\begin{array}{l}\text { littéralement : indien brûlé, peau un rien } \\
\text { foncée, cheveux frisés et traits « ordinaires » }\end{array}$ \\
\hline Jabao & $\begin{array}{l}\text { Clair qui pourrait passer pour blanc si ce n'étaient } \\
\text { les traits de son visage et son type de cheveu }\end{array}$ \\
\hline Pinto & $\begin{array}{l}\text { Clair avec de nombreuses taches de } \\
\text { rousseur et grains de beauté }\end{array}$ \\
\hline Leche/leche bambí & $\begin{array}{l}\text { littéralement : lait bambi du nom d'une } \\
\text { marque de lait en poudre pour enfants }\end{array}$ \\
\hline Blanco & Blanc \\
\hline Rubio & Blond \\
\hline Gringo & Américain blanc \\
\hline Blanco desteñido & littéralement : blanc déteint \\
\hline Jojoto & Blanc anémique \\
\hline Jinchao & Blanc avec des traits « ordinaires » \\
\hline Amarillo & Jaune ; uniquement employé pour les asiatiques \\
\hline
\end{tabular}

Une autre couleur pourrait encore être ajoutée à cette liste, souvent dite à mi-mot et avec un petit sourire : verde (vert). Le vert représente les dollars américains et ce terme est employé pour désigner les personnes noires bien dotées économiquement ${ }^{8}$.

Ainsi, on voit à quel point le vocabulaire coloriste dominicain est riche en nuances. Il peut toutefois être résumé en trois teintes (blanco, negro et indio) qui furent mises en exergue lors du processus de définition de l'identité dominicaine (ou « dominicanité ») élaborée à partir de la seconde moitié du $19^{\text {ème }}$ siècle (Franco 1997) ${ }^{9}$. Ils sont très fréquemment employés par la population dans les interactions mais aussi dans la sphère administrative ${ }^{10}$.

Blanco fut toujours associé à la figure du colonisateur et à un statut socioéconomique élevé ${ }^{11}$. Ceci fut permis par la stratification sociale coloniale, séparant les maîtres blancs des esclaves noirs. Cette figure coloniale était hautement valorisée sur le

8 Si l'emploi de l'adjectif verde n'est pas généralisé, il dit cependant beaucoup de l'idée que l'argent blanchit les gens et que l'on retrouve dans d'autres sociétés post-esclavagistes, comme en Haïti avec ce dicton « Mulàt pov sé nèg, nèg rich sé mulàt » (le mulâtre pauvre est noir, le noir riche est mulâtre) (Labelle 1978 : 136). Cependant, ce dicton est mis à l'épreuve en milieu populaire où les personnes blanches ne sont pas véritablement assimilées aux personnes noires car beaucoup considèrent que malgré les difficultés économiques, les blancs ont sans doute plus de possibilités de «s'en sortir », de décrocher un bon emploi parce que leur phénotype les avantage (Bourgeois 2005 : 77-78).

9 L'identité nationale dominicaine s'est construite en opposition radicale par rapport à Haïti et ce, sur plusieurs plans : racial (blancs/métis vs. noirs), religieux (catholicisme vs. vaudou) et langagier (espagnol vs. créole), pour ne citer que les principaux. Voir aussi Zaglul (1990) et Théodat (2003).

10 Actuellement, la couleur de peau est mentionnée sur les cartes d'identité ainsi que sur le permis de conduire. Selon Moya Pons (2009 : 148 et suivantes), les premiers recensements de population en RD (de 1920 à1960) incluaient cette donnée qui fut toutefois retirée à partir du recensement de 1970.

11 Par ailleurs, dans l'histoire de la colonisation de l'île, nous pouvons voir une tendance certaine des élites blanches à se marier au sein de leur classe sociale. Les alliances matrimoniales réalisées en dehors de ce groupe étaient rares et, le faible taux de métissage de cette franche sociale s'est si bien maintenu qu'aujourd'hui encore cette classe sociale est de peau plus claire. Au contraire, comme nous le verrons plus loin, les stratégies matrimoniales des classes moyennes et basses sont ouvertes vers le haut (Bourgeois $2005: 87-89$ ). 
plan socio-économique et devint également une référence sur le plan esthétique. Cette valorisation est toujours d'actualité. Dans le champ administratif, blanco s'emploie uniquement pour désigner les personnes blanches.

Negro, associé à la figure de l'esclave, fut dévalorisé pendant toute l'époque coloniale au point que les mulâtres dominicains ont développé un sentiment de honte par rapport à leurs origines africaines (Sánchez Martínez 1997 : 52-53). Par ailleurs, l'oblitération historique des cultures africaines lors du processus de définition de la dominicanité a relégué cette couleur à une place de « terme d'opposition » représenté par la seule nation voisine et première république noire : Haïti ${ }^{12}$. Pendant la dictature (1930-1961), à travers l'éducation, les programmes radiophoniques, la presse, les cérémonies et les discours officiels (Capdevila 1998 : 166 ; de la Rosa 2005), la puissante propagande nationaliste dominicaine a stimulé l'émergence d'un préjugé anti-haïtien aujourd'hui profondément ancré dans les représentations et l'imaginaire dominicains (Zaglul 1990). Ceci a donné lieu à une association sémantique et idéologique entre les termes negro et haitiano expliquant pourquoi l'adjectif negro, comme couleur de peau, est, aujourd'hui encore, très peu employé dans la documentation officielle et réservé aux seuls Haïtiens possédant un titre de séjour dominicain ${ }^{13}$. Les représentations dominicaines concernant les Haïtiens et les noirs sont fortement marquées par des stéréotypes négatifs, comme nous pouvons le voir dans l'extrait suivant ${ }^{14}$ :

Il y a des gens qui disent qu'ils sont sales, qu'ils apportent des maladies, en plus ils sont paresseux, il y a des choses qu'ils ne veulent pas faire et tu ne peux pas compter sur eux, ils font beaucoup d'erreurs. [...] Tu vois, du côté de La Vega [dans le Cibao, région centrale de la RD], il y a de nombreux Haïtiens. Et les gens leur donnent du travail, les laissent entrer dans leur maison. Et quand ils [les Haïtiens] se sentent en confiance, paf! ils les [les Dominicains] tuent et $s$ 'enfuient en courant. Et il est impossible de retrouver les assassins, ces prietos se ressemblent tous [...] Là où vivent des Haïtiens, il y a toujours beaucoup de violence, ce sont des voleurs. (V., Loma de Cabrera, 2011)

Il existe aussi d'autres stéréotypes liés au langage (le créole haïtien est décrit comme un patois) et à la religion. Ainsi, lors d'un conflit concernant un office catholique qui allait être célébré par le responsable de la pastorale haïtienne du diocèse de Mao-Monte Cristi (à la frontière) pour l'association de travailleurs haïtiens des plantations de la région, un groupe de Dominicains du village s'opposa à la célébration, barrant l'entrée de la chapelle et une dame s'exprima en ces termes :

12 A noter également que le 27 février, la République dominicaine commémore son indépendance par rapport à Haïti (1844) et non par rapport à l'Espagne (dont la dernière occupation coloniale s'est achevée en 1865 avec la fin de la Guerre de la Restauration) comme la plupart des pays latino-américains.

13 Plusieurs interlocuteurs dominicains noirs ont fait état du rejet de la part des fonctionnaires administratifs quant à leur demande que soit indiquée sur leurs cartes d'identité la lettre $\mathrm{N}$ dans la case « couleur de peau ».

14 Cet extrait n'est pas issu d'un entretien réalisé avec V., en fait, je réalisais un entretien avec une autre personne de la maisonnée et portant sur la main d'œuvre haïtienne dans la campagne frontalière. V., qui nous écoutait depuis la cuisine, est intervenue dans la discussion pendant quelques minutes. 
Ils ne sont pas chrétiens, ils ne croient pas aux saints et ils veulent les brûler.

C'est pour ça qu'on ne veut pas qu'ils utilisent notre chapelle, parce qu'ils vont la casser! (Une dame, Hato del Medio Arriba, 2008)

Dans le premier extrait, on voit clairement apparaître l'association sémantique entre les termes haitiano et prieto, employé pour souligner une noirceur plus prononcée. Et si les attributs personnels et structuraux (Goffman 1975 : 12) ici décrits semblent se rapporter uniquement aux Haïtiens, on les retrouve cependant dans bien d'autres descriptions concernant les personnes noires en général. Par ailleurs, face à des interlocuteurs aux phénotypes différents mais présentant des signes sociaux (habillement, etc.) équivalents, les gens ont tendance à les hiérarchiser socialement et à leur attribuer certaines qualités en fonction de la couleur de chacun (Bourgeois 2005 : 76-80).

Enfin, le terme indio succéda à l'expression «blancos de esta tierra » [blancs de cette terre] utilisée par les mulâtres dominicains pour occulter leurs origines africaines et se défendre de la discrimination socio-raciale. Il fallut attendre 1917 pour que le terme indio soit employé afin de désigner un groupe de personnes (Moya Pons 2009 : 139-140), quelques années après l'apparition de l'indigénisme romantique dans le pays (Franco 2005). Par ailleurs, le processus de définition de la «dominicanité »a privilégié l'emploi de ce vocable qui allait dans le sens de la mythification de l'origine autochtone, marquant de cette manière une rupture nette avec le passé esclavagiste de la population. Les premiers recensements nationaux (1920, 1935, 1950 et 1960) ne mentionnaient pas encore le terme indio comme couleur de peau et privilégiaient l'emploi de mestizo [métis] dont l'occurrence augmenta significativement pendant toute la période dictatoriale afin de présenter la nation comme étant à majorité blanche (Moya Pons 2009 : 150-151). Actuellement, le terme indio est l'une des trois couleurs de peau inscrites sur les documents d'identité ${ }^{15}$ et le recours fréquent à ce vocable pour la description et l'auto-désignation montre bien la réticence des Dominicains à se percevoir, s'identifier et à être reconnus comme mulâtres. Indio est donc socialement très valorisé car il sert d'euphémisme pour décrire les phénotypes proches du mulâtre et éviter l'usage du terme « noir ».

\section{Du vocabulaire coloriste : usages et fonctions}

En République dominicaine, quelle que soit la classe sociale, la pratique de décrire les gens par un terme référant à leur phénotype s'est maintenue. Ceci apparaît très clairement dans cette description familiale :

Chez moi, nous sommes quatre enfants de nos deux parents. Il y a trois indiecitos : maman, un de mes frères et une de mes sœurs. Et nous sommes trois morenitos: papa, mon autre frère et moi. Mais il y a aussi ma demi-sœur du côté de ma mère, elle est indiecita mais plus foncée que maman, elle a de bons cheveux [cabello

15 Les trois couleurs disponibles dans le fichier informatique utilisé pour la création des cartes d'identité sont : indio (I), blanco (B) et negro (N). Dans la grande majorité des cas, ce sont les fonctionnaires administratifs qui déterminent eux-mêmes la couleur de leur interlocuteur, le choix de la lettre à apposer est donc sujet aux représentations de chaque fonctionnaire et, à l'exception des étrangers, c'est la lettre I qui est inscrite sur la carte d'identité. 
bueno] et une petite bouche. Papa a eu deux autres fils avec une femme qui vit à Mao. L'un est moreno, il a de mauvais cheveux [cabello malo] mais meilleurs que les miens, il sont frisés, et l'autre frère est chocolate, grand et a des traits fins. (L., Dajabón 2009)

Le vocabulaire coloriste est également utilisé dans les interactions quotidiennes comme les interpellations, les salutations, les conflits et les moments de tendresse. En ce qui concerne les deux premiers types d'interactions, les gens tendent à se référer aux autres personnes par des euphémismes (moreno/a, indio/a, rubio/a, par exemple), en aucun cas les termes negro et blanco ne sont utilisés. En voici un exemple mettant en scène un accompagnateur de transport public [cobrador], chargé d'encaisser le prix du voyage et de trouver des passagers. Le minibus roule puis s'arrête, le cobrador qui se tenait debout dans la porte ouverte descend du véhicule et part à la rencontre de potentiels voyageurs. Il en interpelle plusieurs qui répondent " non » de la tête et s'arrête devant une dame qui semble hésitante :

Hé toi, Morena! Où vas-tu? Monte, monte! On part tout de suite. Viens Morenita, on t'emmène chez toi! Et cette indiecita, c'est ta fille? Allez, montez, il y a de la place pour toutes les deux. (Santo Domingo 2009)

Ce type de scène se donne à voir dans bien d'autres situations comme les rencontres dans la rue, les interactions avec des guichetiers par exemple, etc. Le locuteur cherchant à attirer l'attention du destinataire a recours à des termes d'adresse correspondant « à l'ensemble des expressions dont [il] dispose pour désigner son ou ses allocutaires » (Fracchiolla et al. 2010 : 3). Or, les seuls éléments ici disponibles sont des éléments visibles : la couleur et les traits corporels. En choisissant des euphémismes et des diminutifs (morena/morenita, indiecita) - véritable stratégie de politesse (selon l'expression de Cini 2010:5 - indépendamment de la couleur « effective » du destinataire, le locuteur diminue la distance sociale entre eux, instaure « une relative égalité de statut entre les participants » (Fracchiolla et al. $2010: 3$ ) et assure par là le bon déroulement de l'interaction. Au contraire, le recours à d'autres mots tels que negro/a, prieto/a, dans le cas d'un destinataire à la peau noire, met en péril l'interaction. De par les stéréotypes qu'il charrie, le terme negro/a employé comme terme d'adresse est perçu, tant par celui qui le reçoit que par celui qui le prononce, comme une injure : « Si on te dit negro, pour beaucoup de gens, c'est une insulte » (J., Santo Domingo 2004). Or, quand on sait que par ailleurs, les termes negro/a, prieto/a, moreno/a utilisés dans la famille et au sein du couple sont porteurs d'une haute charge affective ${ }^{16}$, on peut s'interroger sur la permanence des stéréotypes négatifs liés à cette couleur et de là, poser la question de leur transmission.

16 Cet emploi du vocabulaire coloriste correspond au quatrième type d'interactions, celui des moments de tendresse. Sans vouloir m'attarder plus longuement sur ce registre car cela dépasse les limites de cet article, je dirai simplement que les termes negro, moreno (et leurs dérivés et diminutifs) prononcés au sein d'un couple symbolisent l'amour que les gens se portent. Ils reflètent également une forte connotation érotique et sexuelle, jouant alors sur d'autres stéréotypes comme la puissance sexuelle liée à la couleur de la peau (Bourgeois 2011). Le paradoxe que porte cette couleur était déjà présent dans les « fantasmes d'hypersexualité et de lascivité [...] projetés sur la noirceur » dans l'Occident chrétien (Bonniol 1995 : 190). Voir également à ce sujet: Beckles (2004). 


\section{Identifier et discriminer : l'apprentissage des stéréotypes}

Pour aborder la question de la transmission et de l'apprentissage des stéréotypes, je partirai d'une courte vignette ethnographique. La scène se déroule en août 2011 à Loma de Cabrera. Je raccompagne chez elle B., 10 ans, qui est venue me saluer. Nous passons devant une maison dont la porte ouverte nous permet d'apercevoir une fillette haïtienne nettoyant le sol. A la vue de B., avec qui elle jouait encore deux ans auparavant, la fillette sort en souriant et hèle cette dernière. Ignorant l'appel, B. me dit :

- Je ne joue plus avec cette prieta, elle est Haïtienne et je ne joue pas avec les Haïtiens.

- Mais c'est une petite fille comme toi.

- Elle n'est pas comme moi, tu ne le vois pas? Elle est Haïtienne et les Haïtiens sont sales. Je n'aime pas les prietos. (B., Loma de Cabrera, 2011)

Nous retrouvons dans cet extrait l'association sémantique « noir = Haïtien » ainsi que le stéréotype de la saleté17. Or, quand j'ai connu B. en 2007, elle ne distinguait pas socialement entre ses camarades de jeu et portait encore moins ce type de jugement. Que s'est-il donc passé dans ce laps de temps?

L'apprentissage des stéréotypes raciaux, ou « savoirs sociaux », « implique le déploiement de mécanismes cognitifs puissants et spécialisés de nature à faciliter l'apprentissage » (Hirschfeld 1999 : 16). Ainsi, l'enfant apprend d'abord à catégoriser son environnement, son regard s'affine puis, petit à petit, il parvient à distinguer les gens, notamment en discriminant entre certaines caractéristiques phénotypiques. A ce stade, les différences que le petit enfant perçoit ne possèdent pas encore de signification sociale, culturelle et/ou de classe. Pour comprendre comment ces perceptions physiologiques vont progressivement se combiner à des valeurs sociales et culturelles, je me pencherai sur les facteurs sociaux qui favorisent l'incorporation, par les enfants, de ces significations et, partant, des stéréotypes. Je développerai ici quatre médiateurs spécifiques : le langage (termes d'adresse et adages), les injonctions sociales, l'iconographie et les mises en scène quotidiennes de l'altérité.

\section{Termes d'adresse et dictons}

Dans la première partie de cet article, j'ai souligné combien l'emploi de termes d'adresse coloristes pouvait, dans certaines situations, être perçu comme une injure. Or, ces insultes n'ont pas uniquement cours dans les interactions entre inconnus. Elles peuvent aussi être prononcées par des membres de la communauté ou du voisinage, essentiellement dans les interactions conflictuelles. Au sein de la famille, les insultes à caractère coloriste sont l'expression d'une colère utilisée pour punir ou offenser. Une mère à son fils de 8 ans qui vient de renverser un baquet d'eau : " Maudit prieto ! Regarde ce que tu as fait ! Viens ici, je vais t'en coller une ! Prieto du diable... » (D., Valle Nuevo, 2008). La référence au vocable negro dans des situations de conflit est très

17 La saleté est souvent citée comme attribut des Haïtiens ; elle peut être reliée à la question de l'impureté elle-même régulièrement évoquée dans les commentaires relatifs au sang et au danger de mélanger les sangs, j’y reviendrai. 
fréquente et l'enfant apprend rapidement que ses mauvaises actions entraînent presque automatiquement l'emploi d'une insulte coloriste.

Par ailleurs, les valeurs négatives attribuées au terme negro/prieto sont renforcées par l'emploi de nombreux dictons populaires qui constituent le mot negro en objet de moquerie et de mépris. Ces adages surgissent dans les conversations les plus anodines comme les réponses aux salutations d'un voisin, les commentaires de type rumeurs, les ragots. En voici quelques exemples :

\begin{tabular}{|c|c|c|}
\hline Dicton & Traduction & Signification \\
\hline $\begin{array}{l}\text { Sólo me falta encontrarme } \\
\text { con un negro. }\end{array}$ & $\begin{array}{l}\text { Il ne manque plus que } \\
\text { je croise un noir. }\end{array}$ & $\begin{array}{l}\text { Se dit quand la journée } \\
\text { a été mauvaise et } \\
\text { que le «pire » peut } \\
\text { encore arriver. }\end{array}$ \\
\hline $\begin{array}{l}\text { El negro o lo hace a la } \\
\text { entrada o a la salida. }\end{array}$ & $\begin{array}{l}\text { Le noir, ou bien il } \\
\text { le fait à l'entrée, ou } \\
\text { bien à la sortie. }\end{array}$ & $\begin{array}{l}\text { Se dit pour souligner } \\
\text { que les noirs commettent } \\
\text { toujours des erreurs. }\end{array}$ \\
\hline $\begin{array}{l}\text { Negro que aprende } \\
\text { latín, tiene un mal fin. }\end{array}$ & $\begin{array}{l}\text { Le noir qui apprend } \\
\text { le latin aura une } \\
\text { mauvaise fin. }\end{array}$ & $\begin{array}{l}\text { Se dit pour souligner } \\
\text { la supposée incapacité } \\
\text { des noirs à s'instruire. }\end{array}$ \\
\hline $\begin{array}{l}\text { El negro es comida } \\
\text { de puerco/perro. }\end{array}$ & $\begin{array}{l}\text { Le noir est nourriture } \\
\text { pour le cochon/chien. }\end{array}$ & \\
\hline $\begin{array}{l}\text { El negro es como las } \\
\text { guineas, que siempre } \\
\text { tira para el monte. }\end{array}$ & $\begin{array}{l}\text { Le noir est comme } \\
\text { les pintades, il } \\
\text { s'échappe toujours } \\
\text { vers les montagnes. }\end{array}$ & $\begin{array}{l}\text { Se dit pour souligner } \\
\text { que la personne commet } \\
\text { souvent des méfaits. }\end{array}$ \\
\hline
\end{tabular}

L'association faite entre ces attributs personnels (la propension à l'erreur et la malhonnêteté) et la couleur noire, permet de renforcer et de transmettre les stéréotypes négatifs. Il existe également d'autres associations du noir avec des éléments qui n'appartiennent pas au champ des attributs personnels mais bien à des classes d'objets et aux espèces animales et végétales ${ }^{18}$, comme le montrent les deux derniers dictons. On retrouve aussi ce même type d'associations dans les commentaires portant sur la force physique des Haïtiens associés à des animaux. En parlant ainsi, comme le signale Vaes (2006: 6):

[...] people show a consistent tendency to reserve the human category for their own group and to attribute a lesser degree of humanity to outgroups.

En outre, le contexte de l'énonciation (ton, attitude de l'énonciateur, gestes) vient immanquablement renforcer la connotation négative de ces termes coloristes. Tout un

18 Voir aussi les expressions langagières : ¡Mira tú, goma de carro! ¡Aceite quemado! [Eh toi ! Pneu de voiture ! Huile brûlée !]. La couleur du pneu et celle de l'huile brûlée renvoient ici à la noirceur de la personne. 
discours s'élabore donc autour de ces expressions, qu'elles visent les personnes à peau foncée ou les immigrés haïtiens. Parlant de la discrimination que subissent les Haïtiens dans son pays, L. nous dit :

Etre negro c'est être plus pauvre, le noir c'est le Haütien et le Haïtien n'est pas instruit, il est fait pour travailler durement. Les gens font cette assimilation. (L., Santo Domingo, 2004)

Cette dévalorisation des personnes noires est également véhiculée à travers certaines expressions langagières liées à des injonctions sociales.

\section{Injonctions sociales et couvertures}

Nous avons jusqu'ici particulièrement porté notre attention sur la couleur de la peau, premier critère sur lequel se base un individu pour identifier et catégoriser les autres personnes. La langue employée par ces « autres » constitue un autre de ces critères. J. et Y., deux enfants nés d'un couple haïtien et vivant dans un hameau dominicain m'ont expliqué que leur mère les battait s'ils parlaient le créole ${ }^{19}$ : «Quand des copains [haïtiens] viennent à la maison, on ne peut pas parler le créole avec eux parce que si Maman nous entend, elle crie et elle part chercher la ceinture » (J. et Y., Valle Nuevo, 2008). De même, certaines personnes de la zone frontalière, qu'ils soient Dominicains ou Haïtiens, tendent à cacher le fait qu'ils savent parler le créole - ou veulent l'apprendre - parce que les gens se moquent d'eux, les interpellent par les vocables negro ou haitiano. Par ailleurs, la langue créole est appelée « patois » et « haitiano », par les Dominicains. De cette manière, ils effectuent une hiérarchisation des langues : le créole apparaît alors comme une langue inférieure à la leur et elle est connotée péjorativement.

Il existe également d'autres critères visibles que l'enfant apprendra à reconnaître en « éduquant son regard " (Escallier 2009) : l'apparence physique de manière générale (traits du visage, types de chevelure, coiffure), la posture du corps et l'habillement. Si ces éléments peuvent paraître plus anodins, ils seront toutefois perçus comme essentiels pour la distinction entre Dominicains et Haïtiens en cas de similarité phénotypique des individus. On ne marche pas, on n'a pas le même port corporel et on ne s'habille pas de la même manière selon que l'on est Haïtien ou Dominicain. Ceci est particulièrement visible dans les milieux populaires ${ }^{20}$ et particulièrement vrai en ce qui concerne la gent féminine. Ici interviennent alors les injonctions sociales, c'est-à-dire des remarques de l'entourage qui visent à l'adoption d'un comportement considéré comme efficient, c'est-à-dire permettant la non-confusion entre negro et indio ou encore Haïtien et Dominicain. Ces stratégies de « couverture »(Goffman 1975), sur lesquelles je

19 On peut interpréter cette scène comme la tentative d'une mère d'occulter les origines de ses enfants vivant en République dominicaine mais on peut également la rapprocher d'une certaine dévalorisation, en Haït, du créole en faveur du français, langue privilégiée en dehors de la sphère familiale comme signe de distinction de classe (Labelle $1978: 82$ ).

20 Les immigrés haïtiens de classes moyenne et haute sont plus difficilement différenciables des Dominicains de même statut économique, les différences quant à l'habillement tendent à disparaître, ceci résultant du processus de distinction (dans le sens bourdieusien du terme) d'avec les classes populaires. 
reviendrai, visent également à diminuer ou gommer un autre stigmate, la pauvreté, omniprésent dans les lieux où j'ai effectué mon travail de terrain.

Ainsi, la présentation esthétique de soi fait l'objet de nombreuses recommandations. Les postures des enfants, apprises culturellement (Losonczy 1983 : 48), font l'objet de commentaires tant de la part des adultes que d'autres enfants plus âgés. "Ne t'assieds pas comme ça, ce sont les femmes haïtiennes qui s'asseyent ainsi ${ }^{21}$ » sont autant de petites phrases, prononcées à l'envi, qui vont permettre à l'enfant de distinguer - par la posture du corps - le Dominicain du Haïtien, lui enseigner à quel groupe il appartient et lui transmettre certains stéréotypes liés à l'autre groupe.

Autre élément faisant l'objet de nombreux commentaires : la coiffure. Celle-ci semble constituer un élément important de présentation et donc de distinction. La mère et dans certains cas le voisinage vont se charger d'inculquer aux enfants - et plus particulièrement aux filles - le type de coiffure socialement efficace. Cela passe d'abord dans la distinction entre pelo bueno et pelo malo : le bon cheveu étant lisse, le mauvais cheveu étant crépu. Et les mères, chargées de peigner quotidiennement leurs filles, de leur rappeler : " J'en ai marre de coiffer ta tignasse [cabello malo], va te faire défriser !». La coiffure est également un indicateur du milieu socio-économique duquel on vient ou de son origine nationale. « J'ai dit à Maman de ne plus me faire des tresses, car ce sont les Haïtiennes et les negras qui portent des tresses, parce qu'elles ont de mauvais cheveux » (B., Loma de Cabrera, 2011).

D'autres injonctions ont trait au choix du partenaire amoureux, lequel est présenté, par la famille, comme devant être plus clair que soi afin de mejorar la raza [améliorer la famille].

Il y a toujours quelqu'un de la famille qui te dit " ne te marie pas avec un noir, il faut maintenir la famille [mantener la familia] », c'est-à-dire qu'il faut se marier avec une personne blanche, belle, avec de bons cheveux, de préférence un étranger. Mais si c'est quelqu'un d'ici alors qu'il soit du Cibao [région nord du pays], blanc et beau. (E., Santo Domingo, 2004)

Ces recommandations sont généralement peu suivies, néanmoins elles transmettent certains stéréotypes liés à la couleur. Ainsi épouser une personne plus claire que soi est vu comme « une manière de s'élever socialement. Souvent, chercher un conjoint qui soit plus clair de peau induit de chercher quelqu'un qui aura fait des études, qui aura une bonne profession » (L., Santo Domingo, 2004). Lorsque l'injonction familiale de no dañar a los nietos [ne pas abîmer les petits-enfants] n'est pas suivie et qu'un enfant à la peau foncée naît, il peut arriver qu'il soit rejeté par l'entourage familial, de même qu'un enfant plus clair recevra généralement une attention plus grande de la part de son entourage et sera plus valorisé que d'autres enfants plus foncés de la famille.

21 En région frontalière, la figure féminine haïtienne la plus connue (et donc par rapport à laquelle il faut se distinguer) est celle de la vendeuse qui s'assied généralement sur les talons sans poser les fesses par terre, les pieds légèrement écartés et posés à plat, et les genoux ramenés à hauteur de la poitrine. 


\section{Iconographie}

Ces stéréotypes liés à la couleur de peau sont également transmis par l'iconographie, présentant des mannequins blancs ou très clairs dans la plupart des publicités et magazines, y compris les revues locales. Les mannequins noirs ou métis sont relégués à une place subalterne, tant au niveau du produit qu'ils représentent, que dans la manière dont ils sont mis en scène (Escallier 2009 : 156). Certaines publicités jouent ouvertement la carte du phénotype et l'association de celui-ci avec des stéréotypes négatifs, pour vendre leurs produits. Dans les livres pour enfants, tout comme dans les caricatures politico-sociales publiées dans la presse, les Haïtiens sont présentés avec des grandes bouches, des nez épatés, cherchant la majeure partie du temps à tirer profit de leur statut d'immigrés, malhonnêtes et parlant mal l'espagnol.

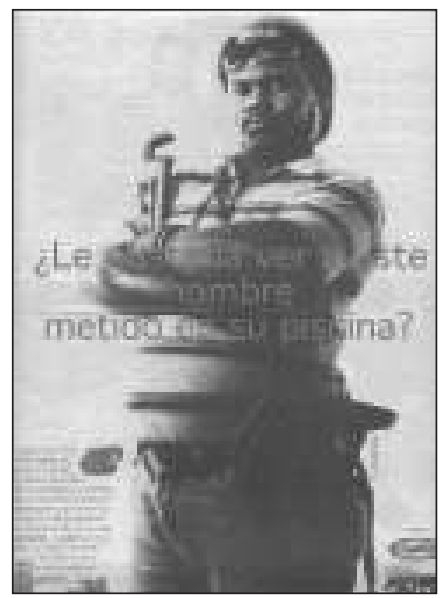

Publicité pour une quincaillerie publiée dans un quotidien dominicain, 2005.

"Cela vous plairait-il de voir cet homme dans votre piscine? Bien sûr que non!

A la quincaillerie $M C$, vous trouverez des pompes submersibles, [etc.].

Placez n'importe quel accessoire de la gamme JACUZZI dans votre piscine ou votre spa et vous ne verrez plus la tête (et d'autres choses) $d u$ plombier».

Caricature d'Harold Priego publiée dans le quotidien national Hoy, le 25 mars 2011. Deux Haïtiens mendiant dans une avenue de Santo Domingo et parlant un espagnol approximatif:

«Tu mendies auprès des jeeps et moi, auprès des Mercedes ».

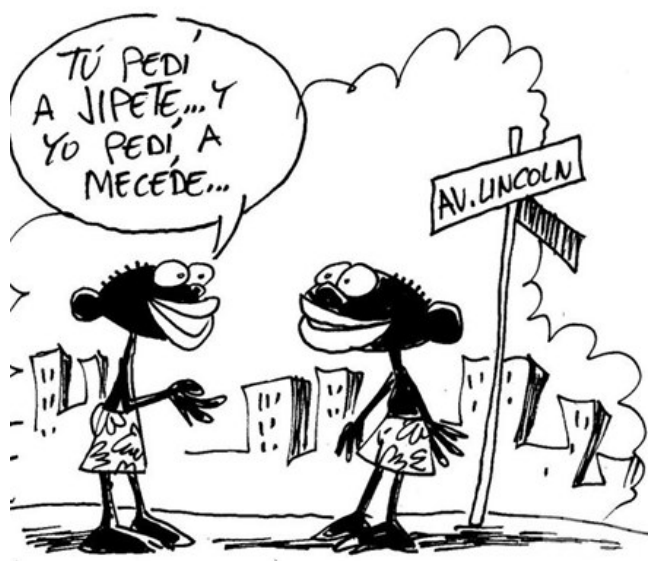




\section{Mises en scène de l'altérité}

Enfin, parmi les médiateurs identifiés comme transmettant les stéréotypes liés aux couleurs, il y a également les mises en scène de l'altérité. Rendons-nous dans une école de la zone frontalière dominicaine, dans le village de Restauración où, peut-être encore plus qu'ailleurs dans le pays, l'identification à la nation fait l'objet d'un surinvestissement social. Ainsi, pendant le mois de la patrie ${ }^{22}$, les écoles primaires et secondaires répètent des saynètes commémorant la guerre d'indépendance dominico-haïtienne. Les écoliers sont amenés à représenter les différents acteurs qui ont participé à cette guerre : les pères fondateurs de la patrie et la créatrice du drapeau national dominicain, d'une part et les soldats des troupes haïtiennes, d'autre part. La représentation de ces saynètes a lieu le 27 février, dans le salon principal de la maison communale, en présence des autorités locales (síndico et regidores), des autorités scolaires, paroissiales, de la fanfare du village et des élèves des différentes écoles. Ce qui nous intéresse ici ce sont les critères dont résulte le choix des écoliers pour représenter ces différents acteurs. Outre le fait d'élire les élèves ayant des capacités à jouer, les rôles sont distribués dans l'école selon l'origine nationale ou la couleur de peau. Or, dans les écoles de la frontière, il y a de nombreux enfants haïtiens. C'est parmi ceux-ci que seront choisis les acteurs des troupes haïtiennes. Et si d'aventure il n'y a pas assez d'écoliers haïtiens pour tenir ces rôles, l'instituteur fait son choix parmi les enfants noirs. Les rôles dominicains sont distribués exclusivement aux Dominicains, à condition qu'ils soient indios. La mise en scène de ces représentations est simple : les deux groupes s'affrontent, les acteurs des rôles dominicains portent d'élégants costumes et déclarent leur amour à la patrie tandis que les acteurs des rôles haïtiens sont vêtus de guenilles et restent muets pendant presque toute la représentation, se conformant de cette manière à la « définition sociale » (Goffman 1973 : 80) des Haïtiens en République dominicaine. Quant aux autres élèves restés dans le public, ils tendent à huer les écoliers jouant les troupes haïtiennes.

Ces «petits riens » du quotidien : la manière dont se donnent les punitions ${ }^{23}$, les petites remarques de la famille et du voisinage, les bavardages des enfants entre eux et ce qui peut sembler comme un détail - comme la distribution des rôles d'une représentation scolaire - «[modèlent $]$ la pensée enfantine et [préparent $]$ le terrain à ce que l'on retrouvera plus tard » (Daréoux 2007 : 90). Or, pendant ce processus d'apprentissage des stéréotypes négatifs, les enfants prennent également conscience de leur appartenance « à l'une [des] catégories sociales, [et celle-ci] ainsi que les propriétés qui y sont attachées, deviennent l'objet de valorisation » (Poulin-Dubois et Serbin $2006: 284$ ).

22 Le mois de la patrie démarre le 26 janvier (date de la naissance de l'un des pères fondateurs de la nation dominicaine) et s'achève le 27 février avec la célébration de l'indépendance dominicaine d'avec Haïti ( $c f$. ci-dessus). Pendant cette période, le pays tout entier arbore le drapeau dominicain, par ailleurs vendu aux carrefours des grandes avenues ou distribué gratuitement dans la presse.

23 Par ailleurs, une étude du SJRM (2005) a montré combien les instituteurs avaient recours à des injures raciales pour souligner les erreurs et les fautes commises par certains élèves. 


\section{Valorisation du « nous », dévalorisation des « autres »}

Cette valorisation du « nous » passe par la dévalorisation des « autres ». Ces « petits riens », tout comme l'usage des appellatifs negro et prieto ne sont pas anodins, ils instaurent, dans le cas des injures, une relation hiérarchique entre le locuteur et le destinataire, le premier « [ posant] dans son énoncé, l'identité [du second]》 (Charaudeau 1992 : 579, cité par Martinez 2010 : 2). En recevant l'appellatif negro/a, le destinataire se voit assigner un phénotype qu'il ne se reconnaît pas nécessairement, d'une part, et qui le rattache à un exogroupe, les noirs descendants d'esclaves assimilés aux immigrés haïtiens hautement discriminés par la société dominicaine en général ${ }^{24}$, d'autre part. De plus, en rappelant le stigmate de la noirceur, les termes coloristes employés insistent également - comme c'est le cas des lieux où j'ai effectué ma recherche - sur le stigmate de la pauvreté, perçue comme inhérente à la condition d'immigré haïtien. Être désigné par les termes d'adresse negro/prieto équivaut à être identifié comme Haïtien et se voir assigner les stéréotypes correspondants. Ceci a des conséquences importantes sur la construction de l'identité des personnes noires. En ce sens, comme le propose Butler, l'injure peut être qualifiée de performative. Par ailleurs, « le discours ne reflète pas simplement une relation sociale de domination ; il décrète [...] la domination, et devient ainsi le moyen par lequel la structure sociale est ré-établie » (Butler 2004 : 45), soit une prolongation des relations sociales coloniales, une réaffirmation des supposées hégémonie hispanique dans l'identité collective dominicaine et supériorité dominicaine dans les relations dominico-haïtiennes.

Si nous soutenons que l'insulte est performative, alors celle-ci renouvelle « la séparation entre les normaux et les stigmatisés » (Dayer 2005 : 316). Or, quand on sait que l'idéologie nationaliste ainsi que les stéréotypes liés au terme noir ont donné lieu à une séparation mentale nette entre Dominicains et Haïtiens, entre indios/blancos et negros, nous pouvons nous poser la question suivante : quels sont les effets de ces associations sur un individu dont le phénotype le rattache au(x) groupe(s) stigmatisé(s)?

La distinction effectuée par Goffman entre discrédités et discréditables arrive à point nommé pour comprendre les réactions des personnes concernées. La personne discréditée est celle dont le stigmate est visible et connu de tous tandis que la personne discréditable est celle dont le stigmate n'est pas directement visible mais qui peut tomber dans la catégorie des discrédités. Il est alors possible pour une personne d'occulter les éléments qui pourraient la discréditer afin d'appartenir (même temporairement) à un autre groupe (Goffman 1975 : 57-58). Or, dans un contexte où le regard affirme la présence noire, où les variations phénotypiques sont certes variées mais d'une certaine manière répétitives, il s'agira pour un nombre important de personnes de se maintenir

24 Comme signalé dans l'introduction, les Haïtiens font l'objet de nombreuses discriminations au quotidien (Wooding et Moseley-Williams 2004) : sur les lieux de travail, dans les écoles mais aussi sur le plan légal puisque la règle du ius solis n'était pas appliquée aux enfants nés de parents haïtiens. Condamnée par la Cour Interaméricaine des Droits de l'Homme, la République dominicaine a modifié l'article référant à la nationalité (désormais applicable en vertu du ius sanguinis) lors de la révision de la Constitution en 2009. Par ailleurs, la Junta Central Electoral a déposé une résolution (012-07) permettant de suspendre temporairement l'expédition d'actes d'état civil supposés obtenus de manière irrégulière, avec effet rétroactif. Sur la discrimination institutionnalisée, voir MUDHA (2000) et Perdomo Cordero (2006). 
dans le groupe des Dominicains ou indios. C'est ce que Goffman appelle les couvertures (Goffman $1975: 123$ et suivantes).

\section{Couvertures et stratégies sociales}

Pour beaucoup de Dominicains, leur stigmate est porté à la connaissance d'un nombre conséquent de personnes. Dès lors, chez les "discréditables », il faut, pour éviter le discrédit, avoir recours à des couvertures c'est-à-dire des procédés servant " à détourner l'attention furtive [portée] au stigmate. [...] [à dissimuler] la réalité à ceux qui l'ignorent [et] à l'adoucir pour ceux qui la connaissent » (Goffman $1975: 123-124)$.

Dans les lieux où j'ai travaillé, deux stigmates sont donc à gommer : la noirceur et la pauvreté. Certes, il est plus difficile de modifier son phénotype sauf en essayant de se déplacer à un autre rang dans l'éventail des gradations raciales. Ainsi, en ce qui concerne les femmes, elles auront principalement recours à deux types de stratégies. L'une est la modification de l'apparence, dont j'ai montré qu'elle fait l'objet d'injonctions sociales. Les femmes travailleront leur chevelure afin de la présenter comme plus lisse et donc éventuellement passer de morena à india. Les couleurs des yeux (utilisation de lentilles de contact), la peau (crèmes éclaircissantes) ainsi que le nez et la bouche (modification par opération chirurgicale) se verront eux aussi modifiés afin de présenter une apparence plus blanche. L'habillement fera également l'objet d'une grande attention, car permettant non seulement d'éloigner de soi le stigmate de la noirceur mais aussi le stigmate moins perceptible, la pauvreté.

Les noirs ressentent un complexe d'infériorité et au moment de se vêtir, ils ont l'habitude de se mettre des beaux vêtements, de belles choses. [...] Ils ont l'impression que les vêtements peuvent les aider à se sentir égaux aux blancs. Il y a toujours une forme de complexe parce que le blanquito est le préféré. (V., Santo Domingo, 2004)

De cette manière, en ayant une bonne présentation, critère ô combien déterminant dans l'obtention d'un emploi, on efface le stigmate du lieu d'où l'on est originaire. Par ailleurs, ces « métamorphoses de l'apparence » (Bonniol 1995) permettent de se distinguer des Haïtiens, dont, je l'ai montré ci-dessus, la posture corporelle, la coiffure et l'habillement sont reconnaissables. Cette attention portée à l'apparence physique est tout aussi déterminante dans « le positionnement social des individus [...] d'où également la mise en place d'une gestion sociale de cette transmission héréditaire » (Bonniol 2006 : 2). Ainsi, même si les injonctions familiales ne débouchent pas toutes sur le choix d'un partenaire amoureux plus clair qu'elles, les jeunes femmes conservent en mémoire un certain idéal phénotypique supposé leur permettre la non-transmission de leur phénotype stigmatisé et leur propre blanchissement par celui de leur descendance, d'une part, et l'assurance d'une situation socio-économique meilleure, d'autre part. Les stratégies matrimoniales des mulâtres sont donc idéalement ouvertes vers le haut et fermées vers le bas, même s'il existe une porte de sortie puisque, comme le dit l'adage, " l'argent blanchit ». Il en sera généralement de même pour les blancs. 


\section{Conclusion}

Comme nous venons de le voir, la question de la couleur et son lien avec la question haïtienne sont bien présentes en République dominicaine. Or, quand certains secteurs de celle-ci - intellectuels, associations ou ONG - émettent une critique par rapport au racisme ambiant et/ou dénoncent la discrimination envers les immigrés haïtiens, ils sont qualifiés d'anti-patriotiques. De même, des représentants de l'Etat, certains dignitaires religieux et des intellectuels nient violemment les rapports portant sur ces sujets et émanant d'organismes internationaux (ONU, Amnesty International, etc.) qui sont alors accusés de diffamation et de tentatives « de dépouiller le pays de sa souveraineté » (Diario a Diario 2011). Ces rapports font également l'objet de larges débats dans la presse et ils sont commentés dans l'espace public. L'argument utilisé pour rejeter ces critiques est presque toujours le même : une population résolument métissée, recourant aux termes coloristes dans des petites phrases galantes destinées à séduire (piropos $)^{25}$, ne saurait être raciste. Or, parler en ces termes, c'est donner une image lisse de la nation, c'est vouloir cacher « ces aspects de l'identité culturelle qui gênent ou embarrassent quelque peu vis-à-vis de l'extérieur » (Herzfeld 2007 : 4). Ainsi, l'aspect métissé de la culture dominicaine est mis en avant et particulièrement visible dans les espaces touristiques. Dans les conversations, nombre de personnes évoquent la question de la couleur noire et celle de l'immigration haïtienne dans des termes semblables à ceux employés par V. quand elle dit « Il y a des gens qui disent qu'ils sont sales », reconnaissant d'une certaine manière que ce type de jugements existent tout en s'en distanciant : il ne faudrait pas que quelque commentaire puisse porter atteinte à l'image de la nation dont l'interlocuteur est aussi l'un des représentants. Cette énergie, déployée pour répondre aux « accusations » et qui laisse à penser qu'on touche à quelque chose relevant de l'intimité culturelle (Herzfled $2007: \mathrm{X})$, sert aussi à cacher que l'identité nationale dominicaine s'est construite sur une identité raciale blanchie en opposition à Haïti, et que les stéréotypes liés à la couleur noire, omniprésents au quotidien, ont été intégrés par une large partie de la population et pèsent lourdement sur la construction des identités individuelles.

\section{Références citées}

Beckles, Hilary, 2004. « Sexe et genre dans l'historiographie de l'esclavage caraïbe », in M. Diouf et U. Bosma (sous la direction de) Histoires et identités dans la Caraïbe. Trajectoires plurielles, 121-139. Paris : Karthala.

Benoist, Jean et Jean-Luc Bonniol, 1994. « Hérédités plurielles. Représentations populaires et conceptions savantes du métissage », Ethnologie française 24 : 58-69.

Bonniol, Jean-Luc, 1992. La couleur comme maléfice. Une illustration créole de la généalogie des « Blancs » et des « Noirs ». Paris : Albin Michel.

-, 1995. « Beauté et couleur de la peau. Variations, marques et métamorphoses », Communications 60 : 185-204.

25 Exemples de piropos coloristes : ¡Pero qué morena que está buena, me imagino que es pura candela! (Quelle belle morena, j'imagine que c'est une vraie bombe !) ou encore : ;Morena! iQue necesitas señalización! ¿Que con tantas curvas, uno se mata! (Morena! Il te faut des panneaux de signalisation! Avec tant de courbes, on pourrait se tuer !). 
-, 1999. "Le métissage entre social et biologique. L'exemple des Antilles de colonisation française », in S. Kandé (dir.). Actes du colloque tenu à la New York University en avril 1997. Discours sur le métissage, identités métisses. En quête d'Ariel, 55-74. Paris : L'Harmattan.

-, 2006. «Penser et gérer l'hérédité des caractères discriminants dans les sociétés esclavagistes et postesclavagistes », Rives méditerranéennes 24. [En ligne] : http:// rives.revues.org/552.

Bourdieu, Pierre, 1979. La distinction. Critique sociale du jugement. Paris : Les Editions de Minuit.

Bourgeois, Catherine, 2005. Vocabulaire et représentations du métissage en zone urbaine : étude de cas en République dominicaine. Mémoire présenté en vue de l'obtention du grade de Licenciée en Sociologie et Anthropologie sous la direction de A.-M. Losonczy. Bruxelles : Université libre de Bruxelles.

-, 2011. « Vocabulario colorista y performatividad en República Dominicana », Communication lors de la Conférence Internationale The Spanish Caribbean : toward a field of its own du 25 au 27 juillet 2011 à Santo Domingo, organisée par le Dominican Studies Institute (CUNY), l'Instituto Global de Altos Estudios en Ciencias Sociales (FUNGLODE), l'Academia Dominicana de la Historia et l'InterUniversity Program for Latino Research (University of Notre Dame).

Butler, Judith, 2004. Le pouvoir des mots. Politique du performatif. Paris : Editions Amsterdam.

Capdevila, Lauro, 1998. La dictature de Trujillo. République Dominicaine 1930-1961. Paris : L'Harmattan.

Cini, Carlotta, 2010. " L'interpellation : interjections et appellatifs. Une approche contrastive italien/français », CORELA - Numéros thématiques "L'interpellation ».

[En ligne] http://corela.edel.univ-poitiers.fr/index.php?id=1698.

Cunin, Elisabeth, 2003. «La "negra Nieves” ou le racisme à fleur de peau. Regards croisés sur une caricature », Bulletin de l'Institut français des études andines 32 (2) : 237-262.

Daréoux, Evelyne, 2007. « Des stéréotypes de genre omniprésents dans l'éducation des enfants », Empan $1(65): 89-95$.

Dayer, Caroline, 2005. « De l'injure à la gay pride. Construction sociale de la connaissance et processus identitaire ", Cahiers de la section des sciences de l'éducation 107.

De la Rosa, Jesús, 2005. « La educación en los tiempos de Trujillo (2) ». Hoy Digital (Saint-Domingue), 17.09.2005.

Deive, Carlos Esteban, 1979. « Notas sobre la cultura dominicana », Boletín del Museo del Hombre Dominicano 8 (12) : 293-306.

Diario a Diario, 2011. «ONG’s pretenden despojar RD de su soberanía », Diario a Diario (Saint-Domingue), 02.11.2009. [quotidien en ligne] : http://www.diarioadiario.com.do.

Escallier, Christine, 2009. « La construction du regard en anthropologie de l'éducation : une ethnopédagogie de l'image », Arquivos da Memória, 5-6 : 52-165.

Fracchiolla, Béatrice, Nathalie Auger, Claudine Moïse, et Christina Schultz-Romain, 2010. « Interpellation et violence verbale : essai de typologisation », CORELA - Numéros thématiques " L'interpellation ». [En ligne] : http://corela.edel.univ-poitiers.fr/index.php?id=1023.

Franco, Franklin, 1970. Los negros, los mulatos y la nación dominicana. Saint-Domingue : Editora Nacional.

-, 1997. Sobre racismo y antihaitianismo (y otros ensayos). Saint-Domingue : Librería Vidal.

-, 2005. « Remanentes ideológicos racistas de la esclavitud. Santana aplastó a los liberales e inició una purga racista », Hoy (Saint-Domingue), Section « Areíto », 25.08.2005.

Goffman, Erving, 1973. La mise en scène de la vie quotidienne. 1. La représentation de soi. Paris : Les Editions de Minuit.

-, 1975. Stigmates. Les usages sociaux des handicaps. Paris : Les Editions de Minuit.

Herzfeld, Michael, 2007. L’intimité culturelle. Poétique sociale de l'Etat nation. Québec : Les Presses de l’Université Laval. 
Hirschfeld, Lawrence A., 1999. « La règle de la goutte de sang ou comment l'idée de race vient aux enfants », L'Homme 39 (150) : 15-39.

Jamard, Jean-Luc, 1992. « Consomption d'esclaves et production de 'races' : l'expérience caraïbéenne », L'Homme 32 (122-124) : 209-234.

Labelle, Micheline, 1978. Idéologie de couleur et classes sociales en Haïti. Montréal : Presses de l'Université de Montréal.

Losonczy, Anne-Marie, 1983. « Color, rasgos y gestos. Reflexiones sobre el automodelo corporal y la identidad en los afroamericanos », Etnica $19: 47-51$.

Lucena Salmoral, Manuel, 1996. Los Códigos Negros de la América española, UNESCO/ Universidad de Alcalá de Henares.

Martinez, Marie-Louise, 2010. «L'interpellation en classe, entre indifférenciation et différenciation des personnes », CORELA - Numéros thématiques «L'interpellation ». [En ligne] : http://corela.edel. univ-poitiers.fr/index.php?id=1033.

Moya Pons, Frank, 2009. La otra historia dominicana. Saint-Domingue : La Trinitaria.

Movimiento de Mujeres Dominico-Haitianas (MUDHA) (éd.), 2000. El nombre y la nacionalidad como simbología de la existencia. Saint-Domingue : Mudha.

Perdomo Cordero, Nassef, 2006. La discriminación racial en el ordenamiento jurídico dominicano. SaintDomingue : SJRM.

Poulin-Dubois, Diane et Lisa A. Serbin, 2006. « La connaissance des catégories de genre et des stéréotypes sexués chez le jeune enfant », Enfance 58 (3) : 283-292.

Quijano, Ánibal, 2000. « La colonialidad del poder y clasificación social », Journal of World Systems Research $6(2): 342-386$.

Sanchez Martinez, F., 1997. Psicología del pueblo dominicano. Saint-Domingue : Editora UniversitariaUASD.

Schmidt, Nelly, 2003. Histoire du métissage. Paris : La Martinière.

Servicio Jesuita a Refugiados y Migrantes (éd.), 2005. La actitud racial en República Dominicana. SaintDomingue, Coll. « Relaciones domínico-haitianas ».

Théodat, Jean-Marie, 2003. Haïti-République dominicaine : une île pour deux. Paris: Karthala.

Vaes, Jeroen, 2006. "“They” are Less Human than "We” are : Modern Prejudice in Human Terms », Cahiers de l'Urmis, 10-11. [En ligne]: http://urmis.revues.org/index184.html.

Wooding, Bridget et Richard Moseley-Williams, 2004. Needed but Unwanted: Haitian Immigrants and their Descendants in the Dominican Republic. Londres : CIIR.

Wright, Roger, 1993. « Sociolinguistique hispanique (8-11 ${ }^{\text {ème }}$ siècles) », Médiévales 25 : 61-70.

Zaglul, Jesús, 1990. Imaginaire social et identité nationale (Le cas de la République Dominicaine), Thèse de maitrise en Sociologie sous la direction de C. Castoriadis. Paris : EHESS. 
